\title{
A teoria de redes como subsídio para o estudo da colaboração em projetos de pesquisa
}

\author{
Antônio Raphael Brito Meireles ${ }^{1}$, Ingrid dos Santos Vilas Verde ${ }^{1}$, Maria Teresinha \\ Tamanini Andrade ${ }^{1}$ \\ ${ }^{1}$ Instituto Federal de Educação Ciência e Tecnologia da Bahia - IFBA \\ Via Universitária, s/n - Pintaguinha, Simões Filho - BA, 43700-000 - Brazil
}

arb.meireles@outlook.com, vilas.ingrid@gmail.com, tamanini@ifba.edu.br

\begin{abstract}
In this paper we study the collaborative networks of researchers who participate in research projects in an academic community. Methods and indices Network Theory were used for the analysis. The results suggest networks topologically characterized as small world. This means that it is an enabling environment for collaboration, since it favors the articulation among researchers.
\end{abstract}

Resumo. Neste artigo estudamos as redes de colaboração formadas por pesquisadores que participam de projetos de pesquisa em uma comunidade acadêmica. Métodos e índices da Teoria de Redes foram utilizados para a análise. Os resultados sugerem redes caracterizadas topologicamente como mundo pequeno (small-world), gerando um ambiente propício à colaboração, pois favorece a articulação entre os pesquisadores.

\section{Introdução}

A colaboração científica é um tema que vem sendo estudado mais intensamente nos últimos anos, mas não é recente [Andrade et al. 2015, Andrade et al. 2014]. Segundo [Sonnenwald 2006] colaboração é a interação que facilita não só a realização de tarefas, mas, também, o compartilhamento do significado desta tarefa, relacionada a um objetivo maior compartilhado entre dois ou mais cientistas.

O estudo de redes tem suas origens na teoria dos grafos, um ramo da matemática. Assim, uma rede é um grafo constituído de um conjunto de elementos chamados vértices ou nós, que são ligados por outro conjunto de elementos chamados de arestas que fazem conexões com os vértices.

Uma rede social é um conjunto de pessoas ou grupos de pessoas com algum padrão de contato ou interação entre elas [Wasserman e Faust, 1997]. Segundo [Barabási 2003], uma rede complexa é um grafo que apresenta uma estrutura ou propriedades topológicas não triviais, composto por um conjunto de vértices (nós) que são interligados por meio de arestas.

Com os índices da teoria das redes é possível caracterizar uma rede como aleatória, livre de escala ou small world, que são os modelos mais difundidos. Uma rede apresenta o comportamento small world se seu coeficiente de aglomeração médio for 
muito maior que o coeficiente de aglomeração de uma rede aleatória e se seu caminho mínimo médio for comparável com o caminho mínimo médio da rede aleatória correspondente.

Para discutir e caracterizar as relações de colaboração foi utilizada três medidas de centralidade: centralidade de grau, centralidade de proximidade e centralidade de intermediação.

\section{Objetivo}

O objetivo desta pesquisa é estudar as redes de colaboração formadas por pesquisadores que participam dos projetos de pesquisa em uma comunidade acadêmica enquanto parâmetro para compreender como ocorre a colaboração nestas comunidades, identificar o modelo de rede associado e as implicações desse modelo nas comunidades às que pertencem os pesquisadores.

\section{Metodologia}

Analisamos um Programa de Pós-Graduação na área disciplinar da Coordenação de Aperfeiçoamento de Pessoal de Nível Superior (CAPES). A escolha deste PPG deve-se a presença de pesquisas de mestrado e doutorado e ao fato de possuir conceito 5 (cinco) pela CAPES. Os cadernos de indicadores de Projeto de Pesquisa são preparados com base nos dados que os Programas de Pós-Graduação informam anualmente.

Os cadernos foram obtidos no formato PDF e neles foram coletados os dados referentes aos projetos de pesquisa (título, participantes do projeto, período, financiamento e situação).

Estudamos os cadernos de indicadores de quatro triênios (2001/2003, 2004/2006, 2007/2009 e 2010/2012); e o período completo (2001-2012). Cabe ressaltar que foi considerado para análise o período das avaliações trienais e relatórios da coleta CAPES a partir do início das atividades do Comitê Internacional Interdisciplinar da CAPES. Foram criados arquivos em formato TXT para cada triênio e outro para o período completo com os nomes dos pesquisadores dos respectivos projetos.

Utilizamos o software Ucinet para gerar redes no formato Pajek; a seguir foram calculados, com o auxílio dos softwares Ucinet e Pajek, os índices e parâmetros da Teoria de Redes.

\section{Discussão dos Resultados}

Nesta seção apresentamos alguns resultados referentes às redes de Projetos de Pesquisa por triênios e do período total, bem como os índices e os seus parâmetros da Teoria de Redes.

Na Figura 1 apresentamos a rede de Projetos de Pesquisa. Os vértices são os pesquisadores e as arestas são as colaborações dos participantes nos projetos de pesquisa. 


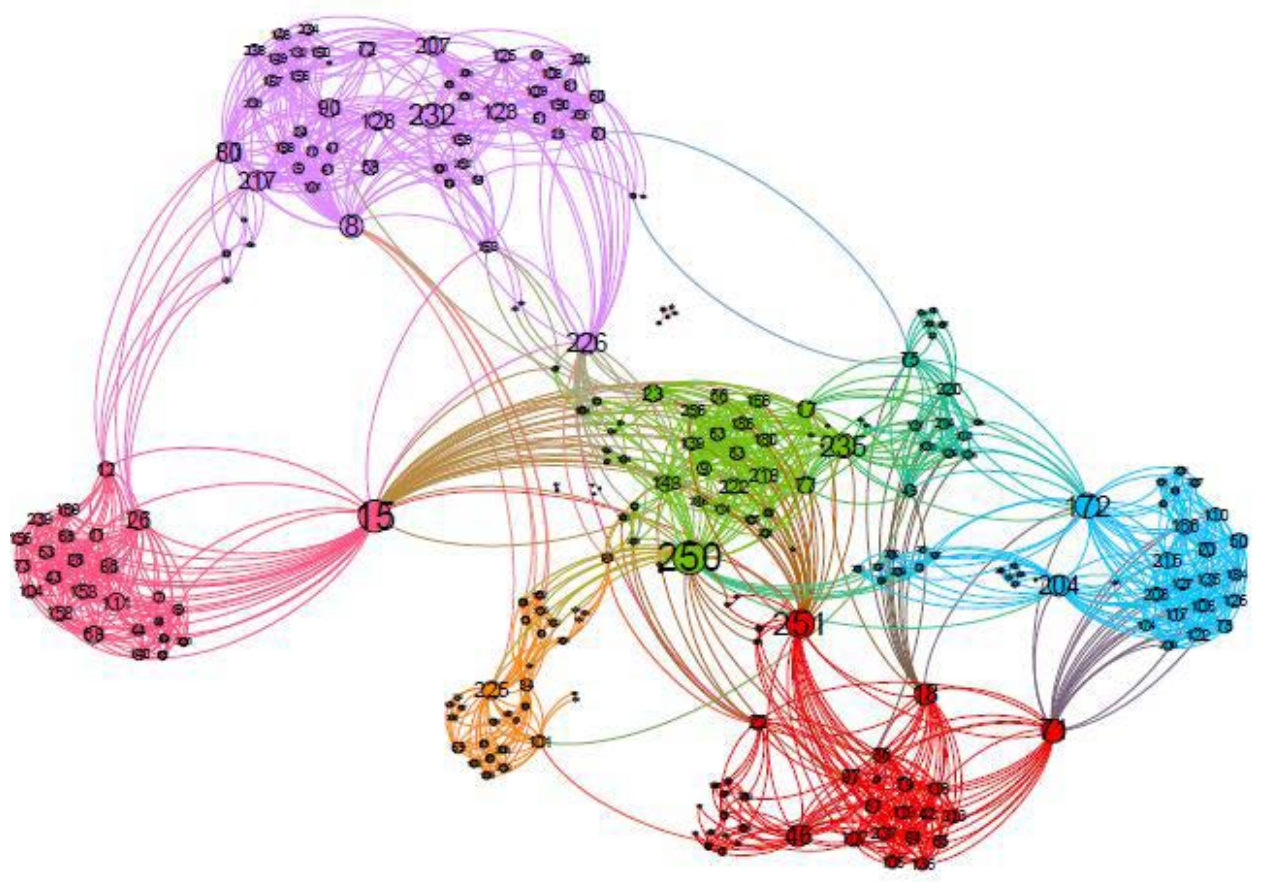

Figura 1. Rede de Projetos de Pesquisa

$\mathrm{Na}$ Tabela 1 apresentamos os índices e parâmetros da Teoria de Redes para Projetos de Pesquisa. O Cr é o coeficiente de aglomeração da rede aleatória e o $L r$ o caminho mínimo médio da rede aleatória.

Tabela 1. Índices de Projetos de Pesquisa

\begin{tabular}{|c|c|c|c|c|c|}
\hline ÍNDICES & TRIÊNIO 1 & TRIÊNIO 2 & TRIÊNIO 3 & TRIÊNIO 4 & $\begin{array}{c}\text { PERÍODO } \\
\text { TOTAL }\end{array}$ \\
\hline Vértices & 13 & 109 & 135 & 135 & 256 \\
\hline Componentes & 11 & 2 & 3 & 8 & 6 \\
\hline Densidade & 0.0256 & 0.117 & 0.060 & 0.081 & 0.051 \\
\hline Diâmetro & 2 & 7 & 9 & 7 & 6 \\
\hline Coeficiente de aglomeração $\boldsymbol{C}$ & 0.000 & 0.876 & 0.883 & 0.873 & 0.833 \\
\hline $\boldsymbol{C r}$ & 0.000 & 0.129 & 0.066 & 0.088 & 0.051 \\
\hline Caminho mínimo médio L & 1.250 & 2.060 & 2.570 & 2.276 & 2.476 \\
\hline Lr & 1.333 & 2.603 & 4.446 & 3.000 & 3.065 \\
\hline Grau Médio & 0.307 & 12.642 & 8.059 & 10.874 & 13.031 \\
\hline
\end{tabular}

As redes estudadas são caracterizadas topologicamente como redes mundo pequeno (small-world). Isso significa que é um ambiente propício à colaboração, pois favorece a articulação entre os pesquisadores. O coeficiente de aglomeração alto $(0<C<1)$ retrata uma situação onde existe uma forte interlocução entre os pesquisadores; o caminho mínimo baixo sugere que o grupo de pesquisadores é ágil em relação ao acesso e contato entre os pesquisadores. 
Nos índices obtidos para as centralidades de grau (CG), de proximidade (CP) e de intermediação (CI) observamos pesquisadores que se destacam. Os docentes P18, P142, P251 e P253 (Figura 1) são os pesquisadores com maior destaque nas redes de colaboração, visto que aparecem em todos os índices de centralidades. Observa-se que a presença desses integrantes é fundamental para o desenvolvimento da colaboração e difusão das informações na rede. Estes pesquisadores que se destacam nos índices analisados são vértices importantes, podendo interagir rápido com os demais $\mathrm{e}$ exercendo controle nesta, formando um núcleo de pesquisadores que fomentam o desenvolvimento e o fortalecimento das redes do programa interna e externamente.

\section{Considerações Finais}

Houve um aumento significativo do número de pesquisadores ao longo dos triênios, logo, ao analisar triênios mais recentes é possível observar maior quantidade de trabalhos sendo desenvolvidos. Com isso é possível constatar que alguns fatores como, maior investimento financeiro e/ou demanda externa ou amadurecimento da comunidade científica ocasionaram maior contingente de pesquisadores atuando e desenvolvendo Projetos de Pesquisa.

As redes obtidas nos dois primeiros triênios possuem características muito comuns. Uma das principais é o fato de terem poucos vértices quando comparadas as redes maiores, como a rede dos triênios 3 e 4 . Isso pode ser interpretado como uma fragilidade momentânea devido ao programa estar iniciando e as redes de colaboração ainda estarem por se formar. A metodologia de análise de redes é uma ferramenta que facilita a identificação da função dos participantes nas redes de projetos de pesquisa.

\section{Referências}

Andrade, M. T. T., Rosa, M. G., Fadigas, I. S., Pereira, H. B. B. (2015). “O papel dos docentes em programas de pós graduação: uma abordagem baseada em redes", In CSBC-BraSNAM, p. 1-6.

Andrade, M. T. T., Braga, P. F., Carneiro, T. K. G., Ribeiro, N. M., Moret, M. A., Pereira, H. B. B. (2014). “Contextualized Analysis of Social Networks: Collaboration in Scientific Communities”, Social Networking, v. 3, p. 71-79.

Barabási, A.L. (2003). "Linked: How everything is connected to everything else and what it means for business, science and everyday life". Plume.

Rosa, M. G., Fadigas, I. S., Andrade, M. T. T., Pereira, H. B. B. (2014).“Clique Approach for Networks: Applications for Coauthorship Networks". Social Networking, v. 3, p. 80-85.

Sonnenwald, D. H. (2006). "Scientific Collaboration", In: Annual Review of Information Science and Technology, Medford, NJ: Information Today, ed. B. Cronin, v. 41, p. 643-681

Wasserman, S., Faust, K. (1997). "Social network analysis: methods and applications". Cambridge: Cambridge University Press. 